\title{
Analysis of Operation of Throttle Micro-cooler at Nitrogen Use with Near-critical Parameters
}

\author{
Dmitry Uglanov a , Dmitry Sarmin, Alina Akulova, Daria Aksenova, Sophia Dostovalova \\ Department of Heat Engineering and Heat Engines, Samara University, 443086, Moskovskoye shosse, 34, Samara, \\ Russia
}

\begin{abstract}
The work of a throttle cooling system with a standard balloon and a throttle cooling system from a cryogenic filling balloon is considered. A comparison between the geometric and hydraulic characteristics of the micro heat exchangers of the two systems is made. The prospects of using the latest system for cooling of photodetector devices (PD) of the infrared range of navigation and research systems for aerospace applications are also shown.
\end{abstract}

\section{Introduction}

In the modern aircraft for aviation and space applications various opto-electronic system (OES) and navigation, research and military equipment (laser rangefinders, rifle scopes, guidance systems, lidar, gyroscopes) are increasingly used [1,2]. One of the devices included in these systems, regardless of their purpose, is a photodetector (PD) of the infrared range, which works effectively only when it is cooled to a temperature level of $80 \mathrm{~K}$ and below. For cooling photo-detectors (PD) IR range throttle systems are widely used as a closed or open cycle. The operation time of the throttle balloon cooling systems depends on the weight of the working fluid and the required cooling capacity.

There is a constructive solution that allows to significantly increase the time of operation of the throttle cooling system and not to overload the balloon with high pressure. So, it is proposed to use the so-called cryogenic filling balloon (patent of Samara state aerospace University № 2163699, Russia) as a part of the throttle cooling system [3,4]. Its feature is cryogenic filling, which allows for the same volume of the balloon and the mass of the refilled working fluid to increase the time of operation of the throttle system by 1,5 times.

\section{Method of calculation}

In this work, the main characteristics of the heat exchangers of the throttling cooling systems with a standard balloon and a balloon with cryogenic filling (universal gas tank) with nitrogen are calculated using a working medium having a temperature of $130 \ldots 135 \mathrm{~K}$ at a pressure of $4 \mathrm{MPa}$ [5]. Figure 1 shows the working cycles and schemes of these systems. In the first case, a standard balloon is used in the throttle system, filled with nitrogen with an initial pressure $p_{0}=30 \mathrm{MPa}$ and a temperature $T_{0}=293 \mathrm{~K}$. In the second case, the above universal gas balloon is used as part of the throttle system.

\footnotetext{
${ }^{a}$ Corresponding author : dmitry.uglanov@mail.ru
} 
Previously performed thermodynamic calculations showed that in case of filling of the balloon with cryogenic products (nitrogen) 30...40hours the equilibrium state of the balloon-working system sets in. The temperature amounts to $T_{0}=130 \ldots 135 \mathrm{~K}$ at pressure $p_{0}=4 \mathrm{MPa}$. In both cases, the initial mass of the working fluid is the same.

The work of the throttling micro-cryogenic installation in the real conditions of use is characterized by the presence of a number of non-stationary modes, "deforming" workflow microcoolers. The main initial parameters of the process - the pressure of there frigerant $p_{\text {input }}$, its temperature $T_{\text {input }}$ and the flow $M$ (or one of these parameters) - are changed not only during the start of the installation, but also in the cooling mode of the object.

The cooling mode with variable flow $M$ is used in micro-coolers with cooling capacity regulators and with "start-up" sections of the heat exchanger, the mode with variable $p_{\text {input }}$ is typical for open cooling systems, and the $T_{\text {input }}=$ varcondition is typical for micro-cryogenic systems, since they usually operate at variable ambient temperature and temperature change in the balloon.

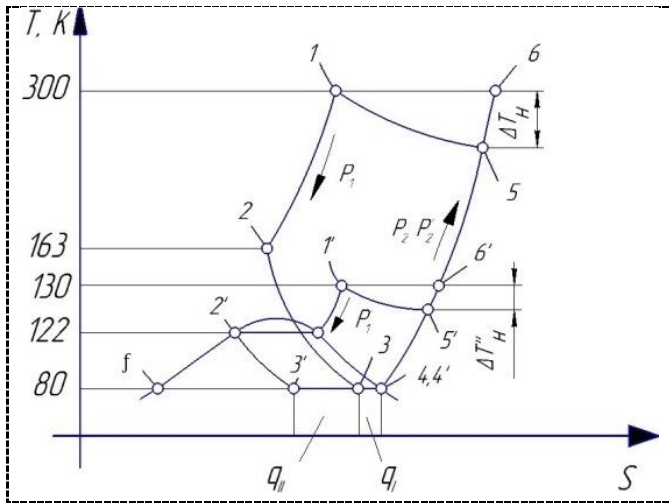

a)
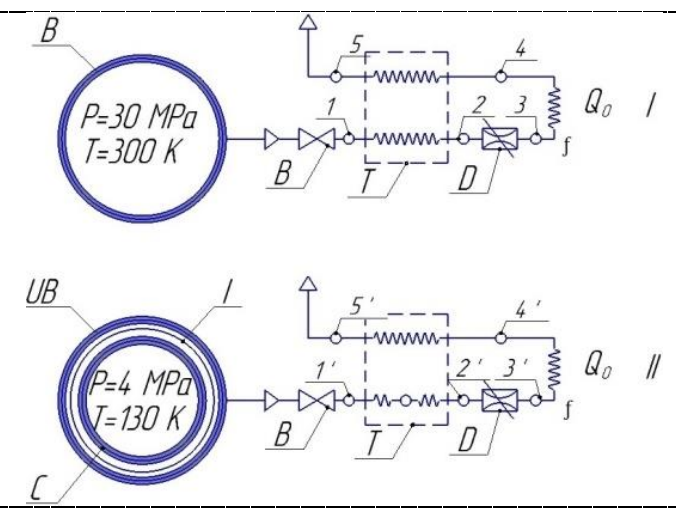

b)

Figure 1. The work of throttle cooling systems: a) the workflow in the T-S diagram; b) - circuit throttle cooling systems: I-conventional balloon system; II - a system with a universal balloon; B -balloon; B -shut-off valve; D -throttle valve; I - thermal insulation; $\mathrm{C}$ - vessel(thermos); UB -universal balloon, $\mathrm{T}$-heat exchanger.

Experience in the development of micro-cryogenic systems has shown [6] that the throttle heat exchanger is characterized by exceeding the available cooling capacity in relation to the thermal load on the system. Micro-cryogenic installation is usually expected to work in extreme conditions, that is, either for the minimum possible in the cooling mode of the working refrigerant pressures and maximum ambient temperatures, or for the start-up period conditions, when it is necessary to provide a relatively short time for the system to go into operation.

To calculate the characteristics of the heat exchanger, a method was chosen for calculating the "excess" cooling capacity $Q$ at a variable flow rate of the refrigerant [7]. In this case, $M=m M_{o}$ (where the exponentm $>1$ ); $Q_{0}^{*}$ - thermal load on the microcooler; Mo is the minimum refrigerant flow (at the beginning of the cooling system with $m=1$ ), $M$ is the actual flow rate.

In the initial mode, i.e. at $m=1$, the temperature pressure at the warm end of the heat exchanger is equal to $\Delta T_{H_{0}}$, for the throttle cooling system with a universal gas balloon $\Delta T_{H_{0}}{ }^{\prime}$ (all letters denoting the thermodynamic parameters for the second system in the future go with a stroke) and is determined by the total heat exchange surface $F_{o}$ and the average integral heat transfer coefficient $k_{o}$ from the high-pressure direct flow to the reverse flow. The temperature of the gas before the throttle is equal to $T_{2_{o}}$ and can be found for the enthalpy of point 4 :

$$
i_{20}=i_{4}+\frac{Q_{0}^{*}}{M_{0}}
$$

If the refrigerant flow rate increases by $\mathrm{m}$ times, the difference $i_{4}-i_{3 j}$ (in calculation $3_{i j}$ - the enthalpy of the working fluid after the throttle D) shall decrease by the same factor.This difference 
increases over time as a result of the removal of the working fluid mass from the balloon at the calculation step $j, i_{4}-i_{3 j}$, necessary to compensate the load $Q_{0}^{*}$, which corresponds to an increase in the gas temperature before the throttle from $T_{2 o}$ to $T_{2 j}$. However, the actual temperature value of $T_{2}$ is determined by the joint action of a number of factors related to change $M$, i.e.an increase of about $m$ times the thermal load on the heat exchanger, an increase in the heat transfer coefficient between the forward and backward gas flows is proportional to $M^{0,7-0,9}$, an increase in the temperature head between the flows due to the growth of $\Delta T_{\mathrm{H}}$ and, to a lesser extent, the change in the heat inflow of the environment, from the gas microcooler and additional cooling of the parts in this zone of the object cryostat.

Thus, at $T_{2}<T_{2 j}$, the surface of the heat exchanger $F_{o}$ can be divided into three zones: $F_{p}$ provides cooling of the direct flow to a temperature corresponding to the constant heat curve, which passes through point 4 (Fig. 1a); $F_{x}$ - necessary for further lowering the temperature of the gas to the maximum permissible value $T_{2} ; \Delta F_{x}$ - "excess" surface, which in principle in this regime microcooler cannot be used. Therefore

$$
F_{0}=F_{p}+F_{X}+\Delta F_{X}
$$

The inequality $\Delta F_{x} \geq 0$ is a condition for the normal operation of the microcooler. The values $m=1$ and $m=m_{\max }$, at which $\Delta F_{x}=0$, correspond to the minimum and maximum refrigerant flow rate, providing the required operating mode of the cooling device; in the range $m$ from 1 to $\operatorname{mmax} \Delta F_{x}$ reaches a maximum, and some other parameters - extreme values. In the heat exchange zone corresponding to the $\Delta F_{x}$, the return flow of the refrigerant contains the liquid phase.

Since the thermal load on the microcooler $Q_{0}^{*}$ is constant, the microcooler leaves the operating mode when the flow value is $M>m_{\max } M o$

$$
M\left(\Delta i_{T}-\Delta i_{\mathrm{H}}\right)<Q_{0}^{*}
$$

$\Delta i_{\mathrm{H}}-$ loss of cold from under exercise.

For the studied mode, the dependence is determined by the $\Delta F_{x}=f(m)$. Heat transfer in different zones of the heat exchanger can be described by equations:

$$
\left\{\begin{array}{c}
k_{X} \cdot \Delta T_{X} \cdot F_{X}=Q_{0}^{*} \\
k_{P} \cdot \Delta T_{P} \cdot F_{P}=Q_{P}=Q_{P X}-Q_{0}^{*}
\end{array}\right.
$$

Here $\Delta T_{X}, \Delta T_{P}$ - mean temperature differences between streams, and $k_{X}, k_{P}$ - medium-integral value of the heat transfer coefficients respectively for the areas of $F_{X}$ and $F_{P} ; Q_{P}=M\left(i_{1}-i_{4}\right)$ - heat load to the area $F_{P}$ of the heat exchanger; $Q_{P X}$ is the same in the area of $F_{P}+F_{X}$.

In accordance with [5], and taking that $\frac{k_{X}}{k_{P}}=z$ and $\frac{k_{P}}{k_{P_{0}}}=m^{\beta}$, the following formulas are used for calculation:

$$
\begin{gathered}
F_{X}=F_{P} \cdot \frac{k_{P}}{k_{X}} \cdot \frac{\Delta T_{P}}{\Delta T_{X}} \cdot \frac{Q_{0}^{*}}{Q_{P}} \\
\Delta T_{X}=F_{0}-F_{P} \cdot\left(1+\frac{\Delta T_{P} \cdot Q_{0}^{*}}{z \cdot \Delta T_{X} \cdot Q_{P}}\right) \\
F_{P_{0}}=\frac{F_{0}}{1+\frac{\Delta T_{P} \cdot Q_{0}^{*}}{z \cdot \Delta T_{X} \cdot Q_{P}}} \\
\frac{Q_{P}}{Q_{P_{0}}}=\frac{M}{M_{0}}=m=\frac{k_{P} \cdot \Delta T_{P} \cdot F_{P}}{k_{P_{0}} \cdot \Delta T_{P_{0}} \cdot F_{P_{0}}} \\
\frac{\Delta F_{X}}{F_{0}}=1-\frac{1}{m^{\beta}} \cdot \frac{\Delta T_{P_{0}}}{\Delta T_{P}} \cdot \frac{\Delta T_{X_{0}}}{\Delta T_{X}} \cdot \frac{\Delta T_{X} \cdot z \cdot m \cdot\left(Q_{P X_{0}}-Q_{0}^{*}\right)+\Delta T_{P} \cdot Q_{0}^{*}}{\Delta T_{X_{0}} \cdot z \cdot m \cdot\left(Q_{P X_{0}}-Q_{0}^{*}\right)+\Delta T_{P_{0}} \cdot Q_{0}^{*}}
\end{gathered}
$$




$$
i_{2}=i_{4} \cdot\left(1+\frac{1}{m}\right)+\frac{i_{20}}{m}-\frac{\bar{z} \cdot \Delta \bar{T}}{m^{1-\beta} \cdot M_{0}} \cdot \frac{i_{1}-i_{2_{0}}}{i_{5_{0}}-i_{1}} \cdot \frac{Q_{0}^{*}}{\Delta T_{0}} \cdot \frac{\Delta F_{X}}{F_{0}}
$$

The heat exchanger of the microcooler is single-row, transverse-counter flow ( $\beta$ is a quantity that depends on the design of the heat exchanger in accordance with the calculated data of $[6] \beta=0,87$ ); the throttle is made in the form of a micro-hole of constant cross-section.

\section{Results of calculation}

In the calculations it is assumed that the temperature along the length of the heat exchanger varies linearly. The hydraulic losses through the heat exchanger channels were calculated in accordance with the procedure [7,8]. Figures 2-6 show the results of calculating the characteristics of the microcoolers from the parameter $m$, which shows the change in the required flow rate to ensure a constant cooling capacity (Table 1).
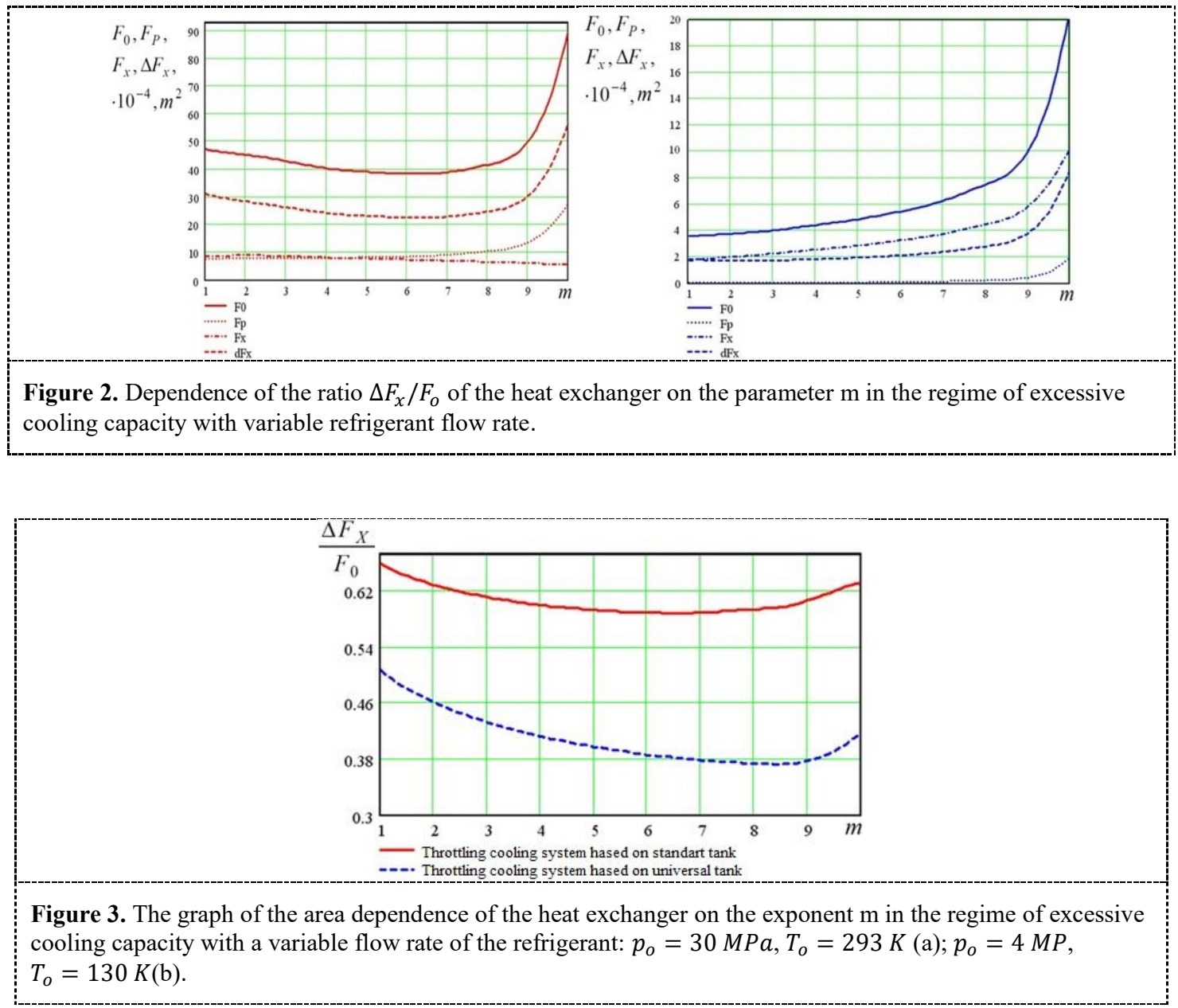

Fig. $2 \mathrm{a}$ and $2 \mathrm{~b}$ show the dependencies of the required surface of the microcooler as a function of the exponent $\mathrm{m}$, which varies during the operation of the balloon throttle system. Comparison of the obtained data allows to make a conclusion that the required heat transfer surface of the microchiller of the throttle system with a universal gas balloon is 5 ... 10 times smaller than that of a similar system 
with a standard balloon. The maximum difference is observed at low flows of the working fluid, i.e. at the beginning of the cooling system operation.

\begin{tabular}{|l|l|l|}
\hline Figure 4. The dependence of the height of the casing of the heat exchanger $H_{\text {case }}$ from indicator $m$ mode of \\
excess cooling capacity with variable refrigerant flow.
\end{tabular}

Table 1. Characteristics of micro coolers depending on parameters $m$.

\begin{tabular}{|c|c|c|c|c|}
\hline \multirow{2}{*}{$m$} & The throttle system with a standard balloon & \multicolumn{2}{c|}{ The throttle system with the universal gas bottle } \\
\cline { 2 - 5 } & $\tau, \mathrm{c}$ & $G \cdot 10^{4}, \mathrm{~kg} \cdot \mathrm{c}$ & $\tau, \mathrm{c}$ & $G \cdot 10^{4}, \mathrm{~kg} \cdot \mathrm{c}$ \\
\hline 0 & 0 & 0 & 0 & 0 \\
\hline 1 & 410 & 3,597 & 630 & 1,140 \\
\hline 2 & 820 & 3,709 & 1260 & 1,276 \\
\hline 3 & 1230 & 3,863 & 1890 & 1,474 \\
\hline 4 & 1640 & 4,072 & 2520 & 2,790 \\
\hline 5 & 2050 & 4,361 & 3150 & 2,136 \\
\hline 6 & 2460 & 4,776 & 3780 & 3,296 \\
\hline 7 & 2870 & 5,416 & 4410 & 4,371 \\
\hline 8 & 3280 & 6,557 & 5040 & 6,580 \\
\hline 9 & 3690 & 9,155 & 5670 & 16,73 \\
\hline 10 & 4100 & 21,012 & 6300 & \\
\hline
\end{tabular}

The dependence of the ratio of the excess surface area of the microcooler to its full surface on the indicator $\mathrm{m}$ is shown in Fig. 3. Comparison of this indicator of both systems shows that for a throttle cooling device with a universal gas balloon requires $60 \ldots 70 \%$ less than the excess surface of the $\Delta F_{X}$. For each curve there is a minimum point $(0,37$ - for a throttle system with a universal balloon; $0,58-$ for a throttle system with a standard balloon), which is due to a change in the heat transfer coefficient during the operation of the throttle cooling system. The change in the heat transfer coefficient is due to the change in the basic thermodynamic and thermophysical parameters of the working fluid at different stages of the cooling system.

The size of the casing along the length is determined by the flow rate of the working fluid and similarly to the surface area of the microcooler varies with an increase in $m$ (Fig. 4).

Fig. 5 shows the dependence of the temperature of the refrigerant before the throttle on the indicator $m$ for two throttle systems is presented.

The temperature in the throttle system with a standard balloon is higher than for the second system at $20 \ldots 40 \mathrm{~K}$. This is objectively due to the difference in the initial temperature of the working fluid in the balloon.

Fig. 6 shows the dependence of the hydraulic resistance $p$ in the channels of high and low pressure of the microcooler on the indicator $m$ is an order of magnitude lower for the throttle cooling system with a universal gas balloon. This result is due to the fact that in the throttle system with cryogenic filling to ensure the required cooling capacity there are significantly lower costs and, accordingly, the speed of the working fluid in the channels of the microcooler. 


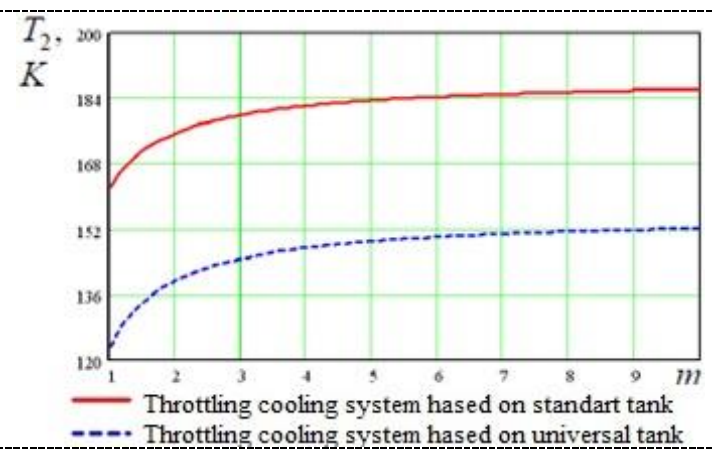

Figure 5. Dependence of the coolant temperature before the throttle on the indicator $m$ in excess cooling capacity at variable flow.

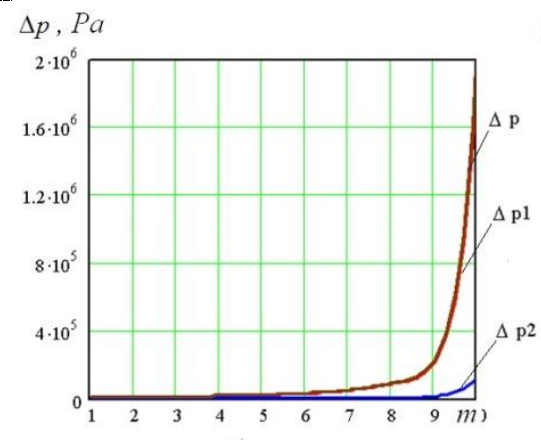

a)

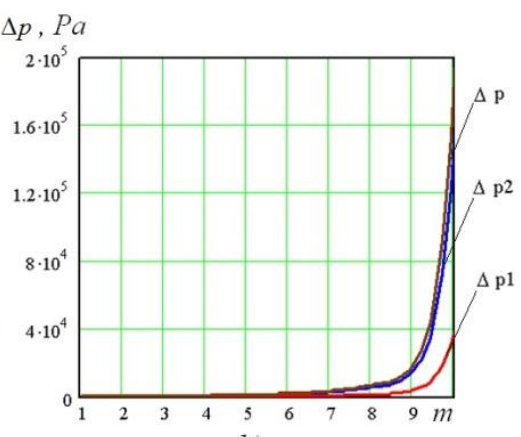

b)

Figure 6. The dependence of the pressure loss $\Delta p$ from the value of $m$ in excess of the cooling capacity with variable refrigerant flow $\left(\mathrm{a}-p_{o}=30 \mathrm{MPa}, T_{o}=293 \mathrm{~K} ; \mathrm{b}-p_{o}=4 M P, T_{o}=130 \mathrm{~K}\right)$.

\section{Conclusions}

Thus, the throttle cooling system with a balloon with cryogenic filling is obtained in 2 times more compact and has a working time of 1,5 times more than the throttle system with a standard balloon at the same initial weight of the working fluid.

\section{References}

1. B.I. Vasiliev, U.M. Manum, Infrared LIDAR for ecology monitoring, 71 (2005)

2. V.V. Protopopov, N.D. Ustinov, Infrared laser location systems, 175 (1987)

3. A.I.Dovgyallo,S.V.Lukachev and others,Fuel tank,Patent №2163699, Russian,MPK 7F17C9/02, 99114577//06

4. E.V. Blagin, A.I. Dovgyallo, S.O. Nekrasova, D.V. Sarmin, D.A. Uglanov, Applied Thermal Engineering Estimation of the energy efficiency of cryogenic filled tank using in different system and devices. 2016. Volume 101, 25 May 2016, Pages 537-544

5. A.I. Dovgyallo, A.P. Logashkin, D.V. Sarmin, D.A. Uglanov, Applied physics: Moscow physical society,Throttle board coolingsystem based on cryogenic filling tank,6, 75-78 (2008)

6. A.K. Grezin, V.S.Zinoviev, Micro cryogenic technique, 232 (2008)

7. V.M. Keis, A.L. London, Compact heatexchangers, 157 (1962)

8. V.V. Sichov, A.A. Vassermann, A.D. Kozlov, G.A. Spiridonov, V.A. Tsimarnii, Thermodynamics nitrogen parameters,(1977) 